\title{
Tematikong representasyon sa pananalig kay Señor Santo Niño: Kolektibong konteksto ng mga deboto
}

\author{
Alvarado, Elsie T. \\ Cebu Normal University, Philippines (alvaradoe@cnu.edu.ph)
}

Received: 5 November 2021

Available Online: 2 January 2022

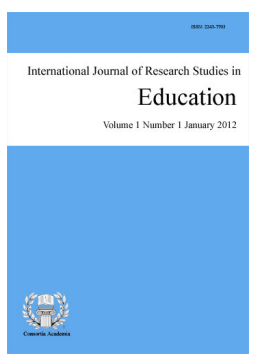

Accepted: 20 December 2021

ISSN: 2243-7703 Online ISSN: 2243-7711

OPEN ACCESS

\section{Abstract}

This study aims to draw up a collective context of faith to Señor Santo Niño from the different experiences of the devotees in Cebu. It specifically answers the representation of faith: meaning of faith, experiences of faith, effects to daily life. Qualitative design was used in the study through katutubong pamamaraan of Enriquez such as pakikipag-usap, pagtatanung-tanong and pakikipagpalagayang-loob so that actual essential data gathered. This study anchored in Filipino Psychology of Enriquez, that refers to the psychology born out of the experience, thought and orientation of the Filipinos, based on the full use of Filipino culture and language. The approach is one of "indigenization from within" whereby the theoretical framework and methodology emerge from the experiences of the people from the indigenous culture. The findings of the study reveal that the devotees conceptualize faith as their beliefs to Santo Niño and it rooted through different miracles that they received towards being an optimistic person in life despite of all challenges experienced. Based on the findings, the study concluded that collectively, the faith by the devotees is connected to all miracles experienced from Señor Santo Niño that lead their strong faith. Thus, the study recommends in the future studies that the relationship of faith and devotion to Señor Santo Niño will be focused.

Keywords: representation, faith, collective, context, devotees 


\section{Tematikong representasyon sa pananalig kay Señor Santo Niño: Kolektibong konteksto ng mga deboto}

\section{Introduksyon}

Ang pananalig sa Senyor Santo Niño ay tumagal at sumulong sa kultura ng Pilipinas sa pagdaan ng mga siglo lalo na sa rehiyon ng Visayas. Ang mga deboto ay naglalakbay taon-taon sa Basilica upang makilahok sa prusisyon at sa kapistahan. Kilala ang mga debotong Cebuano sa matinding pananalig sa batang Hesus, si Senyor Santo Niño na umabot na rin iba't ibang panig ng mundo tulad ng Amerika, Espanya, Italya at sa kasalukuyan sa Indonesia, Korea at Japan. Libo-libong mamamayan mula sa iba't ibang panig ng Cebu ang sadyang pumupunta sa simbahan tuwing Biyernes dahil ito ang araw ng santo maging mga dayuhan. Tuwing unang Biyernes ng buwan ang itinuturing na pinakamahalagang araw ng santo na pinaniniwalaan na naghihimala ang santo at bawat oras ay may misang inilalaan katulad sa linggo. Gayundin ang susunod pang Biyernes ay hindi mahulugang karayom ang simbahan. Matinding pananalig at paniniwala sa santo ang dahilan ng patuloy na pagsamba ng tao kay Senyor Santo Niño. Hindi rin matatawaran ang libo-libong mamamayang sumasamba sa tuwing kapistahan ng santo na tinatawag na Sinulog sa buwan ng Hunyo.

Ang imahen ni Senyor Santo Niño ay hindi lamang tinitingnan ng tao bilang santo kundi parang Diyos dahil hinahalikan, pinupunasan ng panyo at hinahandogan ng bulaklak at iba pang regalo bilang simbolo ng matinding pagsamba. Patunay lamang na sobrang laki ng pananampalataya ng mga tao sa santo katulad sa Diyos. Ayon kay Mercado (1992), personal ang tradisyunal na imahen ng Diyos sa mga Pilipino tulad ng makikita sa iba't ibang debosyong mayroon ang mga Pilipino. Isang katotohanang ang tingin ng mga nagdedeboto sa santo ay isang Diyos dahil maraming hiling ang tinutupad. Likas kung maituturing ang pananampalataya sa santo ng mga Pilipino partikular ang mga Cebuano. Sa katunayan nakasanayan na rin ng mga deboto na nag-aalay ng mga bagay tulad ng laruang pambata, alahas, at iba pang bagay na tanda ng pagsagot ng kanilang hiling. Dulot nito ay bumabalik-balik ang mga deboto upang magpasalamat sa ipinagkaloob na biyaya. Isang kulturang kinagisnan mula noon mapahanggang ngayon ang paniniwala na may himala ang santo sa pamamagitan ng pagtirik ng kandila, paghalik sa santo, pagluhod mula sa pintuan ng simbahan patungo sa altar, at pagrorosaryo sa tulong ng mandadarasal. Nakaugat ang mga kasalukuyang relihiyosong ritwal sa anitismong tradisyon ng kulturang Pilipino (Mercado 1977; Covar 1998; del Castillo et al, 2021). Ang ritwal na hayagang ipinapakita ng mga nagdedeboto kay santo Niño ay ordinaryong senaryo na lamang sa simbahan. Kahit hindi pista o anumang espesyal na okasyon ay di mahulugang karayom ang bulwagan ng simbahan. Ibig sabihin ay bahagi ng pananampalataya ang ritwal ng nagdedeboto sa isang santo bilang pagpapakita ng pagsamba.

Pagsasakripisyo ng mga deboto ay isang pagpapakita ng pananalig sa Diyos dahil nais nitong patunayan na kayang gawin ang lahat ng ginagawa ng Diyos sapagkat naniniwala silang ibibigay ang lahat ng kahilingan kung hinahandugan at pinupuri ang Poon. Ang bawat pagdanas ng tao ng paghihirap ay laging iniaalay sa Diyos. Ika nga ni De Mesa (2010), lagi tayo dapat nagsisimula sa karanasan ng Diyos lalung-lalo na sa Pilipino dahil bahagi na ng kulturang Pilipino ang pananalig sa Diyos. Para sa kanya, hindi nakabase ang pagkakaunawa natin sa Diyos sa "otos" na siyang basehan ng makakanluraning paniniwala bagkus mas angkop ang salitang "dabar" na kung saan binibigyan ng halaga ang ugnayan sa kausap. Ang pagsimba sa linggo ay isang uri ng pagpapahalaga sa Sampung Utos ng Diyos na lubos na pinapairal ng simbahan kung kaya ang mga Cebuano ay nagsisimba sa mga simbahang nais nila. Isa sa mga dinarayong simbahan ang Santo Niño tuwing linggo, sa katunayan maraming nagsisimba na malayo pa ang tinitirhan at maraming simbahan ang dinaanan.

Ang pagmalas ng pagsunod sa Utos ng Diyos ay patunay na malugod na pananalig sa Diyos at lubos na nauunawaan ang pananagutan sa Kanya. Para kay De Mesa (2010), dahil nakabatay sa ating pag-unawa ng karanasan sa Diyos ang pag-intindi natin sa iba't ibang larangan ng pananampalataya, anumang pagbabago ang magaganap sa una ay malamang na magbibigay-daan sa pagbabago sa ikalawa. Magkaakibat at kawing-kawing

58 Consortia Academia Publishing (A partner of Network of Professional Researchers and Educators) 
ang mga ito. Sadyang malaking maitutulong ng pag-unawa sa buhay ng Diyos upang lubos na magalugad ang tunay na kahulugan ng pananampalataya. Kaya nga, sa mga deboto ng santo Niño, hindi inaalintana ang init, layo ng biyahe at sikip ng lugar masilayan lang ang santo. Talagang ganyan ang totoong pananalig sa Diyos hindi naghihintay ng kapalit, ang mahalaga ay masaya at bukal sa kalooban ang pagdarasal.

Madaling magpakatao ngunit mahirap magpakatao na ibig sabihin ay madaling mapaganda ang panlabas na anyo ng tao ngunit mahirap pagandahin ang kaloob-looban nito. Bahagi ng pagdadalisay ng tao sa kanyang sarili ang pananalig sa Diyos. Naging mabuti ang kaibuturang pagkatao ng tao kung may takot sa Diyos at laging sentro sa lahat ng gawain ang Diyos. Ayon kay De Mesa (2010), sa ating pagpapakatao nasasalamin ang ating pakikipag-ugnayan sa Diyos. Ang pakikipag-usap sa Diyos ay idinadaan ng mga Cebuano sa pagsamba sa Santo Niño. Sinasabing instrumento ng Diyos ang santo upang maiparating ang mga dasal dahilang santo ang nagdadasal para sa mga tao. Ang pagtitiwala ng mga Cebuano sa Santo Niño ay walang kapares kung maituturing dahil isang Diyos kung ituring ang santo. Hindi madadaig sa kahit na anong unos at nakikipagbugno pa lalo sa buhay dahil may Santo Niño na handang umalalay. Ang pananampalataya ay ang pananalig at pagtitiwala ng tao bunga ng kanyang pakikipagharap at pagkilala sa Diyos (Miranda 1987).

Napakahalaga sa pananampalataya ang pagkilala at nasasalamin ito sa isang personal na relasyon ng mga tao sa Diyos. Sa pag-analisa ni Miranda (1987), sa pananampalataya, ang "pananam" sa pananampalataya ay maaaring tumukoy sa "lasa." Tumutukoy ang "taya" sa isang sugal na kapag dinagdagan mo ng "pala" ang ibig sabihin ay laging ginagawa. Patunay lamang na maituturing na isang pananalig ang mga ipinapakitang pagsamba ng mga Cebuano sa Santo Niño. Ang kanilang walang humpay na pagpunta lalo na tuwing Biyernes ay patunay na katulad sa Diyos ang pag-ibig at pagsamba ng mga deboto sa batang Hesus. Hindi mikukubli ang tibay ng kanilang pananampalataya dahil kitang-kita sa kanilang mga kilos at gawi. Sabi pa ni Enriquez (1992), ang sikolohiyang Pilipino ay batay sa pagtatasa ng historikal at sosyo-kultural na realidad, pag-intindi sa lokal na wika, pagbunyag sa mga katangian ng Pilipino at pagpapaliwanag nito sa mga Pilipino. Dagdag pa rin niya, ang tuon ng pag-aaral nito ay pagkilala sa pambansang identidad, kamalayan, ugnayang panlipunan, at sikolohiya ng wika at kultura. Ibig sabihin ay saklaw ng pag-aaral ang sining, agrikultura, relihiyon at iba pang gawain sa pang-araw-araw na pamumuhay ng tao. Patunay lamang na sa pag-aaral ng sikolohiya ay kaakibat ang pananalig ng isang tao dahil bahagi ito ng kung anumang relihiyon ang isang tao. Salamin ang pagkatao ng isang tao sa kanyang taus-pusong pananalig at pagpupuri sa Diyos.

Gayunpaman, ang pananalig ay nasusukat lamang sa kung paano ipinapakita ang kaparaanan ng pananampalataya nito bagkus may tinatawag itong kuwento sa likod ng patuloy na pagsamba ng mga deboto kay Senyor Santo Niño. Bawat deboto ay may nakatagong dahilan kung bakit hindi matibag-tibag ang kanilang pagsamba sa Batang Hesus. Wika nina Reyes at Bullecer (2016), ang pamilya ay isa rin sa mga nagpapatibay ng pananampalataya. Anila, ang kahalagahan sa paggawa sana ng pamilya ay maituturing na pangalawang positibong aspeto kasama na rin ng pagkakaroon ng hanapbuhay at pagkakaroon ng mas matibay na pananampalataya sa Diyos. Higit na naging maayos ang hanapbuhay ng pamilya kung dalisay ang pananalig na kung saan pinaniniwalaan din ng mga deboto ng Santo Niño. Mapapansing buong pamilya ay sama-samang pumupunta at sumasamba kay Batang Hesus sa pamamagitan ng pagbibigay ng kanilang mga handog pasasalamat. Ang lahat ng mga natatanggap na grasya ay pinasasalamatan din kaya binalik-balikan talaga ang Santo Niño. Ang mga patron at santo ay binigyang-pugay at respeto para sa sagisag ani na ibinigay ng nakaraang tao at hiilingin na mayroon pang mas masaganang ani para sa susunod pang taon (Reyes at Bullecer 2016; del Castillo at Sarmiento, 2021). Malaki ang epekto ng pagsagot sa mga hiling ng mga deboto kaya patuloy ang kanilang pananalig sa santo.

Bunga nito, ang pag-aaral ay nagnanais na makabuo ng kolektibong konteksto sa pananalig ng mga deboto sa Senyor Santo Niño. Tinitiyak na masagot mabigyang representasyon ang pananalig ayon sa: kahulugan ng pananalig, mga karanasan at epekto sa pamumuhay ng mga deboto. Hangad ng pag-aaral na magkaroon ng malalim na konteksto ng mga deboto ukol sa pananalig sa Senyor Santo Niño upang lubos na mapangalagaan ang halaga ng santo sa buhay ng bawat mamamayang Pilipino. 


\subsection{Mga Layunin Ng Pag-Aaral}

Ang pag-aaral na ito ay nagnanais na makabuo ng kolektibong konteksto ng pananalig mula sa mga deboto ng Senyor Santo Niño. Tinitiyak na masagot ang tema sa representasyon ng pananalig ayon sa: 1) kahulugan ng pananalig; 2) mga karanasan; at 3) epekto sa pamumuhay ng mga deboto.

\section{Metodolohiya Ng Pag-Aaral}

Ginamit ang deskriptibong kwalitatibong disenyo ng pananaliksik upang palarawang masuri ang konteksto ng mga deboto hinggil sa kanilang iba't ibang karanasan sa mga himala ni Senyor Santo Niño. Katutubong pamamaraan ang ginamit sa pagkalap ng datos sa pamamagitan ng pakikipag-usap, at pagtatanung-tanong ni Enriquez (1975). Isinaalang-alang ang mga sumusunod sa panayam: kahandaan ng mga tagatugon; hindi sapilitan; at boluntaryo ang pagtugon sa tanong sa ginawang panayam upang maging palagay sila sa pagsagot.

Nakaangkla ang pag-aaral sa teoriya ng sikolohiyang Pilipino ni Enriquez (1975), ang perspektiba ng mga Pilipino ay nakatuon sa pag-iisip at karanasan ng pagka-Pilipino. Dagdag pa niya, ang sikolohiyang Pilipino ay isang pag-aaral ng diwa, na ang ibig sabihin ng Pilipino ay tiyak na nagbibigay ng maraming ideya na may esensyang pilosopikal at konseptong sikolohikal mula sa isang motibo tungo sa mabuting pag-uugali. Tunay ngang ang pag-aaral ng sikolohiya ay nagkakaroon ng puwang ang mga usapin ukol sa papaano mag-isip ang isang tao at kung papaano naapektuhan ang kanyang mga kilos at gawi sa kanyang pag-iisip. Nagsagawa ng impormal na panayam sa pamamagitan ng pagtatanung-tanong at pakikipagpag-usap sa sampung deboto. Gumamit ng Cebuano sa pagsasalita upang maging natural ang dating ng pakikipag-usap at mas makakapagpahayag sa sarili ang nakapanayam. Naging pamantayan sa pagpili ng sampung tagatugon ang mga sumusunod: 1) nasa apatnapo hanggang animnapo ang edad; 2) katutubong Cebuano; at 3) maraming karanasan sa paghihimala. Limang magkasunod na Biyernes pumunta sa simbahan ang mananaliksik dahil sa araw na ito madalas pumupunta ang mga deboto na namamanata.

Naging instrumento sa panayam ang mga hakbang sa pakikipag-usap bago ang aktwal na pagtatanung-tanong dahil isang mahalagang sangkap ito sa isang katutubong metodo. Una, bumati muna ang mananaliksik na may ngiti sa labi. Ikalawa, upang mas makuha ang totoong sagot ay walang talatanungang ibinigay at pawang ordinaryong pakikipag-usap, at pagtanung-tanong ukol sa karanasan nila bilang deboto kay Santo Niño. Ayon kay Gonzales (1976), sa isang pagtatanung-tanong, walang itinakdang istruktura sa mga tanong at ang pinakamahalagang gabay ng mananaliksik ay ang hangarin ng pananaliksik. Huli, tinapos ang panayam sa pamamagitan ng pasasalamat ng mananaliksik sa mga deboto dahil sa daming testimonya nilang sinabi na bukal-loob ang pagbabahagi. Patunay na masaya sila sa kanilang pananampalataya at ipinamamalaki nila ang pagsamba sa banal na Batang Hesus.

Samantala, upang matiyak ang bawat tema sa representasyon sa pananalig, ginamit na hakbang ang anim (6) na tematikong pamamaraan ni Baun at Clark (2006) na: 1) maging pamilyar sa datos; 2) lumikha ng inisyal na koda; 3) hanapin ang tema; 4) rebyuhin ang mga tema; 5) bigyang-depenisyon ang tema; at 6) pagsulat sa naratibong pagpapakahulugan. Sa tulong ng mga hakbang na ito natiyak ang mga pangunahing representasyon sa pananalig ng mga deboto tungo sa pagbuo ng kolektibong konteksto.

\section{Mga Resulta At Pagtalakay}

Hindi man naging madali ang paghakot ng tugon mula sa sampung deboto ngunit naging maayos naman ang panayam dahil sa ordinaryong pakikipag-usap ang ginawa ng mananaliksik. Ang bahaging ito ay naglahad ng mga tugon ng sampung deboto ukol sa kanilang sariling pagkakaunawa sa pananalig, mga karanasang naganap dito at epekto ng pananalig sa kani-kanilang pamumuhay. Napakalaking tulong ng ginamit na metodo upang maging totoo ang mga sagot ng tagatugon. Ang paghakot ng sagot sa mga suliranin ng pag-aaral ay naging matagumpay. Matutunghayan sa ibaba ang pangkalahatang sagot ng sampung deboto ng Santo Niño. 


\subsection{Kahulugan ng Pananalig}

Ang pananalig at pananampalataya ay magkasingkahulugan lamang. Kaya, sa pag-aaral ay malayang ginamit ang dalawang termino. Sinasabing ang pananalig ay isang paniniwala sa isang Diyos o mga Diyos o sa mga doktrina o mga katuruan ng isang relihiyon. Ibig sabihin ay hindi pareho ang pananalig ng tao na maaaring impluwensya ng paligid o sa mga itinuturo sa bahay at paaralan. Sinang-ayunan ito sa perpektiba ni C.S. Lewis ay naniwalang ang pananampalataya ay isa lamang birtud kung saan ay pinanghahawakan ang mga may katwirang ideya sa kabila ng mga saloobin na salungat dito. Talagang nakakaapekto ng kaalaman ng tao ukol sa kanyang pananalig sa Diyos. Umiikot ito sa kanyang pang-unawa kaya siya naniniwala o nanalig sa Diyos. ang pananampalataya ay walang elemento ng pagdududa. Tunay ngang ang pagtitiwala sa Diyos na mangyari ang nais ayon sa kagustuhan Niya. Naliwanagan ito sa ginawang pag-aaral dahil lumitaw na ang pananalig ng limang deboto kay Santo Niño ay dahil sa himalang naganap sa kanilang buhay. Inisa-isa ang ibinigay na kahulugan ng sampung deboto na humahantong pa rin sa isang himala.

\section{Tema 1. Pananalig bilang pagtitiwala na mangyayari ang inaasahan}

Isang santo na sobrang sinasamba. Hindi inalintana ang layo at pagod basta masilayan lang ang maamong mukha ng santo. Naniniwala ang sampung deboto na ang Batang Hesus ay isang Diyos na tumutupad ng kahilingan. May tiwala naman sila sa iba pang santo ngunit kung ang Batang Hesus na raw ang pag-uusapan ay iba talaga ang hatid nito. Sabi pa nga ng isa sa mga nakapanayam ay bata kasi siya kaya sobrang lambing at bait sa mga tao lalo na sa mga bata. Itinuturing na isang bata si Santo Niño na madaling lapitan kaya matindi ang kanilang pagtitiwala sa kanya. Bilang bahagi ng pananalig sa santo ay pinupunasan, hinahalikan at nakaluhod habang nagdarasal dahil pinaniniwalaan ng mga deboto na makapagpagaling ang santo sa lahat ng uri ng sakit. Malugod na pag-alay sa mga kahilingan sa Batang Hesus ang laging sambit ng mga deboto. Ipinauubaya na sa santo ang lahat ng posibling mangyari sa kanilang mga pasan na suliranin sa buhay. Pakiramdam nila kapag nakapagsabi ng laman ng puso sa santo kay natamo ang kaluwagan sa isip at puso. Iba ang pakiramdam sa tuwing naipalabas ang nilalaman ng kanilang puso lalo na kapag may malaking problema na kinakaharap sa buhay. Sabi pa nga ng isa;

\section{Ipinauubaya ko na kay Santo Niño ang lahat lalo na noong nagkasakit ako. At hindi naman niya ako pinabayaan dahil gumaling ako sa aking karamdaman (DI).}

Batay sa tugon ng deboto, ang matinding pananampalataya sa santo ay malilirip sa bawat salitang binitiwan. Patunay na ang matibay na pundasyon sa pagtitiwala sa kapangyarihan ng santo ang isa sa mga dahilan ng kanyang pagkagaling sa sakit. Habang nagbahagi ang nakapanayam ay hindi maiwasan ng mananaliksik na mapaluha dahil sa sobrang saya na marinig na mayroong ganoon na mananampalataya. Pinatunayan ito sa tugon ng isa pang deboto;

Kukuha ako ng board exam, inihandog ko ito sa kanya sa tuwing Biyernes habang nakikinig sa kanyang novena mass (D5).

Malilirip sa sagot ng deboto sa itaas na ipinagkaloob na niya sa Santo Niño ang lahat para sa kanyang pagkuha ng board exam. Patunay na may matinding tiwala ang deboto na ibigay sa kanya ang hiling sa pamamagitan ng pagdalo sa novena mass. Dahil sa lalim ng kanyang pagtitiwala, naniniwala siya na makapasa sa nasabing pasulit. Bagay na ipinagkaloob talaga nito dahil pumasa siya sa board exam. Malaking pasasalamat ng deboto kaya nangako siya na patuloy na ang kanyang panata sa pagsisimba tuwing Biyernes. Matindi kung ituring ang pananalig ng mga Sebwano na maabot ang mga pangarap sa buhay dahil sa himala ng santo. Dahil dito, mas maraming patuloy na umaasa na ibibigay ang kani-kanilang kahilingan.

\section{Tema 2. Pananalig bilang pag-asa sa buhay}

Sinasabing habang may buhay may pag-asa. Gasgas man ang kasabihang ito ngunit hindi pa rin nalalaos 
Alvarado, E. T.

dahil ito ang laging pinaghuhugutan ng lakas ng karamihang Pilipino lalo na sa panahon ng kagipitan. Madaling sabihin na may pag-asa pa ngunit kung darating ang pagsubok sa buhay ay mahirap palang bigkasin ito. Sa mga debotong napanayam ay bukambibig nila ang salitang pag-asa;

Nananalig ako dahil umaasa na magkaroon ng maginhawang buhay.

Maituturing na inaatang na sa santo ang buhay ng mga deboto ngunit sabi nila ay walang masama dahil hindi naman sila nagpabaya at naghihintay na lang sa himala. Ginagawa naman nila lahat upang makamit ang kanilang mga mithiin sa buhay. Sabi nga ng Diyos ay kumilos ka dahil tutulungan kita. Huwag ipaako sa Diyos ang iyong pananagutan sa mundo. Kailangan mo ring kumilos upang mangyari ang nais mo. Kahit matanda na ang mga tagatugon ay punong-puno pa rin ng pag-asa sa buhay. Hindi raw sila dapat mawalan ng pag-asa dahil nakahanda na raw sila kung ano ang maging bukas. Ang pagtingin sa buhay bilang positibo ay nakakadala sa maaliwalas na pamumuhay. Hindi raw mawawalan ng pag-asa kung may pananalig ka sa Diyos.

Ang Batang Hesus ay itinuturing na ring Diyos ng mga deboto dahil iisa lang daw sila. Ang Santo Niño ay siyang nagdarasal para sa mga tao upang maisakatuparan ang kanilang mga kahilingan. Ang santo ay instrumento para magkakaroon ng pag-asang matupad ang mga dasal. Huwag lang daw sumuko at tumigil sa pagdasal bagkus magpatuloy pa at higpitan pa ang pananalig sa Diyos na baling araw ay masagot ang tanging dasal. Sa katunayan, naging bahagi sa pang-araw-araw na buhay ng deboto ang pagdarasal sa imahen ng Santo Niño. Sabi nga ng tagatugon;

\section{Mayroong akong imahen ni Santo Niño sa altar. Nakaugalian ko ng magdasal sa kanya anumang oras gusto ko. Para bang ito ang pinaghuhuutan ko ng lakas ng loob (D9).}

Bahagi na ng kasaysayan ng bansa ang pagsamba sa mga santo bunga ng kolonisasyon ng mga Kastila. Ang kulturang ito ay minana ng mga Pilipino mula sa mga mananakop at mapahanggang ngayon patuloy na masisilayan ng bawat pamilyang Pilipino. Wika ni Christy (2010), bawat Pilipino ay mayroong imahen ng Santo Niño kahit nakatira pa sa ilalim ng tulay. Isinasaad na ang pananalig sa imahen ay likas na sa bawat Pilipino. Patunay na bahagi ng pananampalataya ng mga Pilipino ang pagsamba sa mga imahen na kung saan masisilayan sa lahat ng simbahang Katoliko. Pinaniniwalaan na ang mga santo ay mga anghel na siyang nagtatanggol sa mga tao. Sa pag-aaral nina Inocian et al. (2016) binanggit na ang totoong karanasan ng deboto sa paghalik o pagpunas ng panyo sa rebolto nito ay naging bahagi na bilang pananalig nitong makapagpagaling ang santo sa lahat ng karamdaman ng taong sumasampalataya sa kanya. Isinasaad na bahagi na sa pananalig ng mga deboto ang pagsamba sa mga imahen na kung saan pinaniniwalaan nilang tumutugon sa kanilang mga dasal at hiling. Kaya, sa mata relihiyong Katoliko Romano, ang mga santo ang magiging daan at instrumento para matupad ang mga dasal at kahilingan.

\section{Tema 3. Pananalig bilang himala ng Batang Hesus}

Sa sampung debotong nakapanayam ay nabuo ang kolektibong kahulugan ng pananalig bilang himala. Ang paniniwalang mangyayari ang mga dasal at pag-asa na matupad ang hiling ay isang pananalig sa himala. Ang himala ay isang katuparan sa isang kahilingan. Naibigay ang kahilingan na hindi inaasahan dahil ibinigay sa hindi inaakalang panahon. Ang limang deboto ay nakaranas ng himala sa Batang Hesus. Ang pagdarasan nang taimtim ay nagbubunga ng kaluwalhatian sa pakiramdam. Sinabi nila na hindi naman sa umaasa sa himala ngunit talagang nangyari ito sa buhay nila. Kung kaya, nananalig sila dahil tunay na natupad ang kanilang dasal at hindi lang puro sabi-sabi.

Ang pagkaranas mismo ng pangyayari ay mas lumalim pa ang kanilang pananalig sa Santo Niño. Ang isa sa mga deboto ay nagsabi na;

Maraming beses na akong nakaranas ng himala ni Santo Niño kaya palagi akong pumunta rito para magpasalamat sa kanyang ipinagkaloob. Hindi ako magsasawang pabalik-balik dito dahil masaya ako sa aking ginagawa. Sa katunayan ay nabanggit niya na mula pagkabata ay palagi 
Tematikong representasyon sa pananalig kay Señor Santo Niño: Kolektibong konteksto ng mga deboto

siyang dinadala ng kanyang ina dahil mahigpit din ang pananalig ng kanyang ina (D6).

Naging bahagi sa kanyang buhay ang pagdarasal na nagpapatibay pa lalo sa kanyang pananalig. Ibig ipahiwatig, bukal sa kanyang kalooban ang pasamba kay Senyor Santo Niño. Dagdag pa niya;

Siguro naimpluwensyahan ako ng aking ina sa pananalig kay Santo Niño dahil namulat akong nasilayan ang mukha ng santo (D6).

Malinaw na ang pananalig ay nakasandig sa kung ano ang karanasan ng mga deboto sa Santo Niño. Naging malalim ang pananalig nila dahil sa totoong kaganapan na nagpapakita ng isang himala.

Samakatuwid, ang kahulugan ng pananalig para sa mga deboto ay bunga ng kanilang matinding pagtitiwala sa Santo Niño sa tuwing ipinagkaloob ang kanilang dasal at hiling at nagbunga ito ng panibagong pag-asa na darating din ang panahon na sasagutin ang hiling na ito. Ang pananalig sa kanila ay tumibay pa lalo dahil sa mga natanggap na himala mula sa Santo Niño na itinuturing nilang pinakamalaking himala sa tanang buhay. Sa kadahilanang hindi inaakalang maganap ang hindi kapani-paniwalang pangyayari sa kani-kanilang buhay.

\subsection{Karanasang Ipinagkaloob ni Senyor Santo Niño}

Bawat tao ay may kanya-kanyang karanasan sa buhay na siyang humuhubog sa ganap na pagkatao. Anuman ang karanasan ng isang tao sa pakikibugno sa buhay ay naging behikulo sa pagtamo ng mga mithiin nito. Ayon kina Reyes at Bullecer (2016), ang karanasan ay ibinahagi at napag-alaman na ito ay nabuo sa katagalan ng pakikilahok at kinalakihan na rin. Pinaniniwalaan na ang karanasan ay bunga ng pakikihalubilo at pakikisama sa kapwa. Malaking impluwensya rin ang kinagisnan o lugar kung saan ipinanganak upang mahubog ang kanyang pagkatao. Isang pagpapatibay ng sarili at pagkamit ng pansariling kalinangan at kaunlaran ang pagkakaroon ng maraming karanasan sa buhay. Hindi matatawaran ang kakaibang karanasan ng mga deboto kay Santo Niño sapagkat naging susi ito upang magbago ang kani-kanilang pananaw sa buhay. Nahuhubog ang karanasan ng mga deboto sa tulong ng samutsaring pagpapakita ng presensya ng santo sa kanilang buhay. Masasabing nagpapakita ng santo kung may naganap sa kanilang buhay na hindi inaasahang mangyari. Pinatutunayan ito sa lahat ng ibinahagi ng mga deboto sa ginawang panayam. Nahahati sa tatlo ang pagtalakay sa resulta ng pag-aaral ukol sa karanasan ng mga deboto kay Batang Hesus.

\section{Tema 1. Karanasang Ipinagkaloob ng Batang Hesus ang kanilang mga kahilingan}

Nangangahulugang sila ay naniniwala na makapangyarihan ang Batang Hesus na parang Diyos dahil lahat ng kanilang dasal at hiling ay natupad. Kaya raw sila pabalik-balik dahil iyon ang kanilang panata kung ibibigay ang kanilang hiling. Gaya na lamang ng pangyayaring ito;

Nagkaroon ng matinding karamdaman, hindi na makalakad ang isa sa mga nakapanayam ngunit nang inialay niya kay Santo Niño ang kanyang sakit ay unti-unti siyang gumaling. Nang siya'y gumaling ay bumalik siya dahil gusto niyang pasalamatan ang santo at bilang ebidensya ay ibinigay niya sa kumbento ang kanyang dalawang saklay na kung saan makikita ito sa museum ng simbahan (D3).

Patunay lamang na ang karanasan sa himala ng santo ay naging dahilan sa patuloy na pagsamba at palaging pagpunta para mag-alay ng dasal. Nagsisilbing panata ito ng deboto upang maipakita ang matinding pananampalatayang handog sa Batang Hesus. Dagdag pa ang sinabi ng kalahok na;

Sabi pa niya hindi siya magsasawang pabalik-balik sa simbahan hanggang buhay pa.

Pagkaroon ng payapa at maginhawang buhay ng mga anak sa kabila ng hirap sa buhay (D8).

Ang patuloy na paniniwala sa santo ay naging bahagi na sa buhay ng kalahok sapagkat kaluwalhatian sa pakiramdam ang kanyang natamasa sa tuwing bumibisita siya sa simbahan. Ang ganitong pakiramdam ay nararanasan lamang niya sa tuwing pumunta sa simbahan upang magdasal. Bagay na natatangi sa lahat sapagkat 
Alvarado, E. T.

hindi nakukuha sa materyal na bagay ang kapayapaan sa buhay bagkus sa matibay na pananalig kay Santo Niño. Dagdag pa ng isang deboto;

Bata pa lang ang isa sa mga deboto hanggang nag-asawa ay bumibisita siya sa santo tuwing Biyernes dahil pakiramdam niya ay magaan ang kanyang kalooban matapos makapagdasal sa santo. Hindi niya inakala na mapagtapos niya ang kanyang mga anak sa pag-aaral at magkaroon ng maayos pamumuhay ang kanyang mga anak(D8).

Ito ang lagi niyang ipinapagpasalamat sa tuwing pumunta siya sa simbahan at higit sa lahat ang pagkakaroon ng malusog na katawan sa kabila ng katandaan. Sobrang tuwa habang ibinabahagi ng isa sa mga nakapanayam ko ang kanyang ideya ukol sa pananalig sa santo. Kakikitaan ng ligalig habang nagsalaysay sa karanasan. Bilang karagdagan ng kalahok, sinabi niyang;

Ayon sa kanya ay tumibay pa lalo ang kanyang pananalig nang binigyan ng pangalawang buhay pagkatapos maaksidente sa sariling sasakyan. Ang akala niya ang patay na siya dahil tumilapon siya nang sobrang layo mula sa sasakyan sa damuhan kasama ang Santo Niño na katabi niya sa lugar na kung saan siya tumilapon. Laking gulat niya dahil maliliit na galos at sugat lang ang natamo sa katawan (D8).

Ang ganitong karanasan ay mas lalong nagpatibay sa deboto na maniwala sa santo at kumapit sa pananalig sapagkat ang aksidenteng iyon ay hindi biro ngunit nalampasan niya. Isa sa dahilan na nakapagpapatibay sa kanyang paniniwala na pinoproktehan siya ng santo ay yaong imahe na nasa harap ng kanyang sasakyan na palagi niyang pinag-alayan ng dasal. Naniniwala ang deboto na dahil sa patuloy siyang nananampalataya, kaya hindi siya pinabayaan. Paliwanag ni Whitehead (2013), ang mga tao at mga relihiyong imahen ay dinadala ang isa't isa sa pagiging inspiradong anyo sa pagiging kakaiba at may ugnayan sa pagkakaranas. Hatid nito ang pagkakaugnay sa pagitan ng tao at paniniwala sa isang pinaniniwalaan ng relihiyong kinabibilangan.

Binanggit ni Bautista (2021), sa kanyang artikulo, naniniwala ang mga deboto na magaling ang Santo Niño sa pagprotekta sa kapaligiran gaya ng natural na kaguluhan, gutom, pagkagaling mula sa matinding karamdaman, o pagpapaunlad sa pinansyal na kalagayan mula sa matinding kalugmukan. Ibig sabihin, likas ang pananalig ng mga deboto sa santo na kung saan naging daan sa pagpapatuloy ng kanilang pagsamba at pananampalataya sa imahen. Tunghayan ang dagdag pa na karanasan ng deboto;

Nagawa pa niyang bumalik sa sasakyan at nagmaneho pabalik sa kanilang tinitirhan habang tinitigan ang imahen ng Santo Niño. Ito ang dahilan kung bakit tuwing Biyernes ay pumunta siya upang magpasalamat himalang natamo (D8).

Malinaw sa salaysay ng deboto na pinaniniwalaan niyang may naganap na paghimala sa pagligtas ng kanyang buhay. Natuklasan sa pag-aaral nina Brion et al., (2018), ang danas sa patuloy na paglaganap sa pananalig na may kapangyarihan ang imahen ay nagmumula sa mga testimonya ng mga deboto. Dagdag pa, ang manipestasyong ito ay napapabilang ang pagtugon sa mga dasal, pagpapakita ng himala, pagpapanatili sa malapit na personal na obligasyon sa pananalig, pagkilala, gantimpala sa lahat ng sakripisyo ng mga deboto, nagbibigay ang tulong sa mga kahirapan, at panindigan sa mga tradisyon (Brion et al., 2018). Batay sa nabanggit na natuklasan nina Brion et al., ang mga testimonya ng deboto ay naghahatid ng iba't ibang manipestasyon na nagbubunga ng kultural na perspektiba na bahagi ng pananalig ang pagsamba sa imahen.

Pinatunayan lamang sa karanasan na ito na ang patuloy na pagdarasal at pananalig ay magbunga ng kabutihan. Ang santo ay palaging nandiyan upang bantayan ka laban sa kapahamakan. Sa kabilang banda, isa sa mga deboto ay nagtamo rin ng biyaya galing sa santo. Anya;

Hindi biniyayaan ng anak ang mag-asawang deboto sa loob ng sampung taon kaya namanata sa santo. Sa kanilang pabalik-balik sa pagdasal sa santo ay nagkaroon ng isang anak na nakapagbubuo sa kanila bilang pamilya (D4).

64 Consortia Academia Publishing (A partner of Network of Professional Researchers and Educators) 
Tematikong representasyon sa pananalig kay Señor Santo Niño: Kolektibong konteksto ng mga deboto

May awa ang Diyos, hindi niya ako pinabayaan at natupad ang aking dasal. Totoo ngang kasabihan na kung magdasal ay huwag mawalan ng pag-asa at maghintay lang dahil darating ang panahon na ibibigay ng Diyos ang hiling. Ang mga pangyayaring nabanggit sa itaas ay maliwanag na may Diyos sa pamamagitan ng santo dahil biniyayaan sila ng grasya. Ang karanasang ito ay nagdulot ng mas matibay na pananalig sa santo. Dahil sa ibinigay ang hiling ay higit na naniniwala at sumasamba kay Santo Niño.

\section{Tema 2. Karanasang Ipinagkaloob ng Batang Hesus bilang Tagagabay}

Sabi nila mayroong tagapagbantay ang tao na isang anghel. Ang pananalig sa kabanalan ng santo na gagabay sa lahat ng ginagawa ng mga deboto ay higit na nakapagpalakas ng loob sa kanila. Inaasa nila ang kanilang desisyon sa buhay kay Santo Niño. Naniniwala sila na nagbibigay ng senyales ang santo kung gagawin ba nila ang kanilang mga iniisip. Sabi nila;

Sa tuwing humihiling ako sa santo ay sinasabi ko sa kanya na ito ang senyales ko kung iyon ay ayon sa kalooban mo. Nagkatotoo naman ang lahat ng iyon kaya naniniwala ako na buhay ang Batang Hesus sa bawat tao. Magtiwala ka lang (D1, D7, D9).

Ang pakiramdam na parang kulang kung hindi nakapunta sa simbahan ng santo ay naibahagi rin nila. Kung mayroon silang kahilingan ay inialay kay Santo Niño at kung anuman ang mangyari ay sana pagkalooban ng patnubay. Gaya ng inaasahan ay nangyari naman ngunit may mga pagkakataon na sinubok talaga ang pananalig dahil may mga hiling na hindi natutupad. Kaya, sa tuwing hindi natutupad ay ipinagdarasal na gagabay ang santo kung ano ang tamang landas na tatahakin. Ang sampung deboto ay nagsabi na kahit may mga dasal na hindi natutupad ay humihiling pa rin na patuloy na maging patnubay sa buhay na tinatahak. Sa kabila ng hindi natutupad na hiling ay mayroong instrumentong ipinagkaloob upang magsabi sa mensahe ng Diyos.

Sumasang-ayon sila na may mga pagkakataon na sumuko sila at tumigil sa pagdarasal at may pag-alinlangan sa kanilang pananaliig dulot ng hindi magandang karanasan ngunit may ipinadalang tagapagsalita ang Diyos. Nang dahil dito ay lalong tumibay ang kanilang pananalig at mas naging kapana-panabik ang kanilang mga karanasan kasama ang banal na Batang Hesus.

\section{Tema 3. Karanasang Ipinagkaloob ng Batang Hesus sa pamamagitan ng pag-ibig na wagas}

Malaking katanungan minsan para sa mga taong naghahanap ng tunay na pag-ibig sa tuwing hindi pa ito nahanap o naramdaman man lang sa tanang buhay. Sa mga nalihis na landas dahil sa iba ang karanasan sa buhay ay ito ang laging laman ng tanong. Kabaligtaran naman sa mga taong punong-puno ng pag-ibig ang puso dahil nababalot ng pagmamahal. Ang pag-ibig ng Diyos ay walang kapantay at kapalit. Hindi matatawaran ang pag-ibig na inialay sa kanyang mga anak. Sabi ng mga deboto ay nakaranas nila ng kakulangan at parang walang saysay ang buhay dahil sa daming dumarating pagsubok. Kahungkagan sa puso ay hindi rin nakaligtas sa kanila dahil sa pakiramdam ay natutulog ang Diyos. Pero may mga pangyayari sa buhay nila na nakapagbabago sa kanilang pananaw. Ang poot ay napalitan ng saya at humantong sa pag-ibig na walang hanggan. Patunay ang sinabi ng deboto na;

Sinabi ng isa sa mga nakapanayam na dumating sa punto na hindi na niya matukoy ang tamang landas dahil puno ng poot ang kanyang puso ngunit may hindi inaasahang pangyayari sa buhay niya (D10).

Sinasabing hindi magbubunga ng mabuti ang poot o galit sa puso dahil maaaring hahantong ito sa kapahamakan. Kaya, ang isang deboto ay naniniwala na mas mainam na maging malawakan ang pagtingin sa mga bagay sa buhay upang mabibiyayaan pa lalo. Sabi ng deboto na;

Isang babae na asawa na niya ngayon ang nagturo sa kanya upang maging positibo sa buhay.

Matagal na niyang hiling na pagkalooban ng babaeng totoong magmahal sa kanya sa kabila ng kanyang kahinaan. Nagkatotoo ang kanyang hiling dahil nakilala niya ang kanyang asawa. 
Matutunghayan mula sa tugon ng deboto ang wagas na pag-ibig. Sinasabing bawat tao ay may ipinagkakaloob ang Diyos na kapares o katuwang sa buhay. Maghintay lamang sa tamang panahon at pagkakataon dahil kusa itong darating na hindi inaasahan bilang sorpresa ng Diyos. Maliwanag na ang kaispang ito ay namumutawi sa tugon ng deboto. Ang ganitong karanasan sa buhay ay maituturing na maswerte o may himala sapagkat ang pagkakaroon ng wagas na pag-ibig sa buhay ng tao ay sadyang pinapangarap ninuman. Gaya ng sabi ng deboto;

\section{Matagal na siyang humiling at nagdasal na makahanap siya ng asawa na maramdaman niyang} ito na talaga ang bigay ng Diyos.

Pansinin na ang matatag niyang pananalig na makahanap ng babae na mamahalin niya habambuhay ay naging daan para matupad ang kanyang hiling. Maliwanag na kung bukal sa puso at buong pagmamahal ang ating minimithi sa buhay ay hindi malayong ipagkaloob ng Diyos.

Sabi ni Fabrega (2009) ang pagbabalik sa mga ipinagkaloob ay nagsasaad ng pagiging mapagpasalamat, pagbibilang sa lahat ng grasyang natanggap, pagkilala sa mga kasiyahan at pagpapasalamat sa lahat ng mga ipinagkaloob. Ang nosyon na ito nagpapatunay na karaniwang reaksyon ng tao ang pagpapasalamat at pagbabalik sa lahat ng kabutihan ng Diyos. Nagbubunga ito ng kaligayahan ng isang tao at pagiging mas positibo pa ng tao (Lyubomirsky 2008). Ang pagkaranas ng mga deboto sa pagkatupad ng mga kahilingan ay naging sanhi ng pagkamatibay pa sa pananalig sa Batang Hesus. Naging instrumento ang asawa niya upang mas humigpit ang kapit niya sa pananalig kay Santo Niño na noon ay malabo pa. Ang pag-ibg nga naman, hahamakin ang lahat masunod ka lamang. Totoo ang kasabihang ito dahil laging may inuutusan ang Diyos para magturo sa mga taong nalihis ng landas. Ang sampung deboto ay natutong magmahal at mahalin pa ang sarili maging ang kapwa dahil sa kabanalang natutuhan sa tuwing nagsimba at nakinig sa mga mabuting salita ng Diyos.

Talagang bahagi sa buhay ng tao ang karanasan. Ito ang tumutulong para tumibay pa ang pananaw at prinsipyo ng tao sa kanyang sarili maging sa buhay. Ang iba’t ibang karanasan ng mga deboto kay Santo Niño na matupad ang hiling, nagkaroon ng tagagabay at nahanap ang pag-ibig na wagas ay daan tungo sa matibay na pananalig sa santo. Lumakas ang kapit sa pananampalataya dahil totoong naranasan ang kapangyarihan ng Diyos sa pamamagitan ng Batang Hesus. Ang imposible ay naging posible sa kanilang buhay dahil nandyan ang santo. Hindi sila pinababayaan ng santo kaya patuloy ang kanilang pagsamba.

\subsection{Epekto sa Pamumuhay ng mga Deboto}

Likas na masayahin ang mga Pilipino sa kabila ng mahirap na pamumuhay ay kayang-kaya pa rin nitong ngumiti at tumawa. Ganyan din ang mga Sebwano na kung saan nakikita rin ng mananaliksik sa sampung deboto na nakapanayam. Kahit may edad na ay masayahin sila habang nagbabahagi sa kanilang buhay. Ang iba't ibang karanasan ng mga deboto dahilan ng patuloy na naniniwala sa kapangyarihan ng Santo Niño at tumibay ang pananalig na ipagtanggol sa lahat ng pagsubok na darating sa buhay. Binanggit ni Bautista (2004), kinikilala ang Santo Niño ng mga deboto bilang bayaning kultural sapagkat naging bahagi na sa buhay ng bawat Sebwano ang pakikilahok sa pagdiriwang at pagdarasal sa kanya. Gayundin, sa bawat pang-araw-araw na gawain nila naging matatag ang kanilang pananalig na kung saan nagbubunga ng pagiging positibo sa buhay, pagkakaroon ng pag-asa at mas lalong naging matatag sa buhay. Unang talakayin dito ang positibong kaganapan sa pamumuhay ng mga deboto.

\section{Tema 1. Positibong dulot ng Pananalig sa Batang Hesus}

Gaya ng inaasahan ay masarap pag-uusapan ang positibong aspeto ng pamumuhay ng tao. Sa buhay ng mga deboto ay masaya nilang ibinahagi ang mga magagandang epekto ng pananalig sa santo. Pagkakaroon ng positibong pananaw sa buhay sa tuwing nakaranas ng problema ay nangunguna sa kanilang ibinahagi. Hindi nila 
Tematikong representasyon sa pananalig kay Señor Santo Niño: Kolektibong konteksto ng mga deboto

ikinakaila na kung maraming masasayang kaganapan sa buhay nila ay katumbas nito ang kalungkutan. Gayunpaman, hindi sila nadadala at naging mas positibo pa ang pagtingin sa buhay. Madalas nilang ginawa kung malungkot ay dinadaan sa tawa ang problema. Naging mas malawak ang kanilang pang-unawa kung may kinakaharap na suliranin sa buhay. Madalas nilang ginawa ay ibinabahagi sa iba at kung may panahon ay ipinahayag sa harap ng santo. Sa katunayan nakasanayan na ang pagtirik ng kandila;

Sanay na sanay na akong magtirik ng kandila, magosaryo at lumuluhod patungong altar ng santo (D2).

Kaya pala maraming nakikita ang mananaliksik noong bata pa na matatandang lumuluhod papuntang altar. Kasunod nito ay naging mas mahinahon at mahaba ang pasensya sa pagdala sa buhay. Madaling bumangon kapag nadapa at idinulog ang problema sa santo. Nakasanayan na ang ganitong gawi na ibinahagi sa santo ang problema. Hindi maiiwasan na hindi pa man nasolusyunan ang isang problema ay mayroon na namang panibago kaya sa ganitong sitwasyon ay mas naging maluwag ang pagtingin ng mga deboto. Ang huli, maraming nangyari na taliwas sa nais ng mga deboto subalit hinarap nila ang mga ito. Nakagawian na ang pagbisita sa tuwing Biyernes at ang miyembro ng pamilya o mga anak sumama na rin sa pagsabi na;

Batobalani sa gugma, sa daang tao palangga. Ang pag-awit ay simbolo ng aming pasasalamat kay Senyor Santo Niño (D3).

Ito ay isang paghatid ng pagsamba sa santo na ibig sabihin, ang santo ay simula ng pag-ibig sa lahat ng tao sa mundo. Dagdag pa nito ay patuloy na pagkaloob ng mga kahilingan na ayon sa mga deboto ay ibinigay ng santo. Kaya, hindi rin sila magsasawang pabalik-balik sa pagsimba at pagsamba sa santo bilang pagbalik sa lahat ng ipinagkaloob sa kanila. Ayon kay Beckett (2015), ang prinsipyo ng pagbalik sa lahat ng mga bagay na ibinibigay sa tao ay nasa dugo na ng tao lalo na sa mga Pilipino. Nangangahulugan ang mga deboto ay nakadikit na sa kanilang buhay ang pananalig sa santo na naging mabuti ang takbo ang kanilang pamumuhay kung nananalig. Ito ay naging sanhi rin sa pagiging positibo sa buhay na lahat ng bagay ay may dahilan at lahat ng problema ay may solusyon.

\section{Tema 2. Pag-asa sa Pananalig sa Batang Hesus}

Hindi perpekto ang tao kaya hindi maiiwasan na makagawa ng pagkakamali. Ang mga deboto ay patuloy na umaasa sa santo. Una, dahil sa pagtitiwala na ipinagkaloob ng santo ang mga dasal ay umaasa na lang sila sa himala. Sabi pa nga nila;

Bahala na si Santo Niño dahil naniniwala ako na ipagkaloob niya ang aking kahilingan sa tamang panahon (D5, D6, D7).

May luha pa sa mga mata nang ibinahagi ng isa sa mga deboto ang kanyang paniniwala nang dahil sa pinaparanas ng santo sa kanya. Pangiti-ngiti at palingo-lingo pa niyang naalala ang nakaraan na hanggang ngayon ay parang hindi pa rin siya makapaniwala na matagumpay ang kanyang negosyo. Kaya, sobrang nagpapasalamat sa santo dahil naging dahilan ito upang kumapit siya noong panahon ng kagipitan. Sinabi niya na;

Matagal man niyang sinagot ang aking mga dasal ngunit sobra-sobra naman sa aking hiling (D1).

Parang ayaw na niyang mabuhay noong panahong iyon dahil sa sobrang kahirapan. Punong-puno pa siya ng utang at hinahabol na siya sa pinagkakautangan. Binabalot na siya ng sobrang insekyuridad sa sarili at muntik nang nawasak ang binuong pamilya. Gayunpaman, dagdag ng deboto na;

Hindi siya sumuko at patuloy siyang nananalig at nagdarasal sa santo na baling araw ay giginhawa rin ang kanyang pamilya (D1). 
Alvarado, E. T.

Sa nabanggit na senaryo ay malinaw na ang kanyang matibay na pananalig na magkaroon ng kaginhawaan at hindi bumitiw at patuloy na umaasa na siyang naging sanhi sa pagkatupad ng kanyang kahilingan. Ipinapakita lang na ang bukal sa loob na dasal sa Batang Hesus ay naging daan sa katuparan ng kahilingan ng deboto.

\section{Tema 3. Pagsisikap sa buhay ng mga Deboto}

Ang pagiging matibay sa pananalig ng mga deboto sa Santo Niño ay siyang nagsisilbing tulay sa pagtuloy na pakikibugno sa buhay. Hindi tumigil sa pagpapasalamat sa lahat ng grasyang ipinagkaloob at nagiging mas matatag pa ang pagsisikap sa buhay. Anila;

Bawat Biyernes akong pumunta at nagtirik ng kandila upang ipagdasal aking pangarap sa buhay. Mula pagkabata ay nakagawian ko ng idaan sa dasal kay Santo Niño ang ninanais ko sa buhay. Parang may kulang kung hindi ako makadalaw sa kanya (D4, D8, D9).

Naglalarawan lang na bahagi na sa buhay ng deboto ang pagsamba at pagpunta sa simbahan upang mag-alay ng dasal sa santo. Ipinapakita lang na matibay ang paniniwala niya sa santo mula pagkabata pa mapahanggang ngayon. Bagay na naghahayag ng nakagawian na niya at ang pagkapit sa pananampalataya ay nagdudulot ng katatagang loob niya sa buhay. Ibig sabihin, mas tumibay ang pananalig ng mga deboto sa Batang Hesus. Binanggit din ng deboto na;

Bawat unang Biyernes ng buwan ay nakagawian ko ng magsimba dito dahil masaya sa pakiramdam kapag nakadalaw ako santo (D3).

Ito ay nagsasaad na bukal sa loob ang pagsimba ng deboto at ang kasiyahan sa loob ay isang patunay na maganda ang epekto ng kanyang pananalig sa santo. Nagsasaad na bukod sa pagdarasal ay may mga ritwal pang ginagawa ang mga deboto bilang pagpapakita ng kanyang pananalig. Nagsisimbolo lang ito ng matatag na pananalig sa santo na nagbubunga ng katatagang loob sa buhay.

Saksi sa lahat ng unos at bagyo sa buhay ang patuloy na pakikipagbugno ng mga deboto laban sa hamon. Hindi kailanman palaging masaya ang buhay, bagkus, napakaraming paghihirap na pagdadaanan tungo sa pagkamit ng mga adhikain. Patunay ang sinabi ng deboto na;

Walang-wala ako noon, dahil sakto lamang ang aking sahod para sa pangunahing pangangailangan ng aking pamilya ngunit nagkasakit ang aking ina. Wala akong pera na pantustos sa kanyang pangangailangan. Hirap na hirap na akong balansehin ang pera para sa aking pamilya at sa aking ina. Dumaan ako sa simbahan ni Santo Niño gaya ng lagi kong ginagawa kapag hindi ko alam ang aking gagawin (D3).

Batay sa sagot ng deboto, pinansyal ang kanyang problema na kung saan hindi niya alam kung saan kukuha ng pera para matustusan ang pangangailangan ng ina na hindi maapektuhan ang pangangailangan ng sariling pamilya. Ang ganitong sitwasyon ay pangkaraniwan sa pamilyang Pilipino sapagkat pinapahalagahan ang kapakanan ng buong pamilya. Malilirip mula sa tugon na naging sandalan ang kanyang pananalig sa santo upang patuloy na magsikap at ipinaubaya niya sa dasal kung ano ang kanyang gagawin sapagkat wala siyang ibang paghuhugutan. Masasabing hindi gaanong mabisa ang kanyang aksyon na ginawa ngunit sa taong walang-wala talaga, lahat gagawin o kakapitan. Sa puntong ito kumapit siya sa pagtitiwala sa santo na makayanan niyang lampasan at masolusyunan ang problema. Sa katunayan, dagdag pa ng deboto;

Pagkatapos kong magdasal, gumaan ang aking pakiramdam. Habang nakaluhod at tinitingnan ang imahe ni Santo Niño, naging magaan ang aking pakiramdam at para bang may liwanag akong nakikita kung ano ang aking susunod na gagawin para masolusyonan ko ang aking problema. Para sa akin, sapat na itong tugon ng santo sa aking dasal na kung agad niyang tinugon ang nilalaman $n g$ aking puso (D4).

68 Consortia Academia Publishing (A partner of Network of Professional Researchers and Educators) 
Tematikong representasyon sa pananalig kay Señor Santo Niño: Kolektibong konteksto ng mga deboto

Inilalarawan sa tugon ng deboto na ang pagkakaroon ng karunungan sa kung ano ang hakbang na gagawin sa kanyang problema ay sapat na dahilan na magsisikap siya na tulungan ang kanyang ina para magkakaroon ng lunas ang karamdaman. Ang nosyon na ito ay pinatunayan ni Hornborg (2006), pinaniniwalaan ng mga deboto na taglay ng imahen ang buhay na tao at tinitingnan ito bilang komunikatibong bagay sa halip na walang buhay na bagay. Patunay ang matatag na pananampalataya ng deboto na kaya niyang ipaubaya ang lahat ng bigat na nararamdaman sa puso ng deboto ay malaking tulong para maibsan kahit papaano ang kanyang kalungkutan. Dahil dito, makapag-isip-isip na siya kung anong dapat gawin para matulungan ang ina sa kabila ng kakulangan sa pinansyal. Ibig sabihin, ang kapanatagang-loob ay siyang natamo at ipinagkaloob sa kanya ng santo. Sapat na masasabing hindi mawalan ng pag-asa na magsikap ang deboto na harapin ang anumang unos sa bahay dahil alam niya na karamay at kasama ang Santo Niño sa laban.

Sa kabuoan, maliwanag na maliwanag na malaki ang epekto sa buhay ng sampung deboto ang pananalig sa Santo Niño bilang sentro ng kabanalan nang sa gayon, patuloy na makikipagbugno at lumaban sa takbo ng buhay. Kinikilala sa popular na kabanalan ang Santo Niño bilang bahagi ng ekspresyon sa tradisyon ng mga ninuno sa pananalig na kung saan ipinapamalas sa pamamagitan ng debosyon sa imahen ni Kristo, Maria at Santo Santo Niño (Roman Catholic Church, 1994). Dahil sa pananampalataya, ang pagiging positibo sa buhay ay nagbubunga ng mas lalong magkakaroon ng pag-asa at pagsisikap na suongin ang hampas at bagyo na dumating at dumarating pa dahil naniniwala sila na hanggang may matibay na pananalig sa Santo Niño, kailanman hindi pababayaan at higit sa lahat mas lalong masungkit ang pinapangarap at minimithi sa buhay, magtiwala lamang sa Diyos.

\section{Konklusyon at Rekomendasyon}

Batay sa mga natuklasan, napatunayan na isang representasyon sa pananalig ang iba't ibang konteksto ng mga deboto na nakabuo ng kolektibong pananaw na bunga ng maraming himalang naranasan kay Senyor Santo Niño at naging dahilan para tumibay ang kanilang pananampalataya na humantong sa sa pagiging positibo sa pananaw sa buhay. Sa katunayan sabi ni Bautista (2010), ang Santo Niño ay hindi lang payak na simbolikong representasyon sa pagiging banal, subalit bilang makasaysayang imahen na may makataong tampok, pinaniniwalaan na mayroong nagsasariling intensyonalidad. Taglay ng sampung deboto ang patuloy na panata tungo sa patuloy na pagtupad ng kanilang mga kahilingan. Habambuhay na nilang kasama ang Batang Hesus dahil hindi kailanman sila pinababayaan sa hirap man o ginhawa. Batay sa nabuong kongklusyon, iminumungkahi na magsagawa pa ng ibayong pag-aaral ukol sa ugnayan ng pananalig at debosyon ng mga deboto ni Senyor Santo Niño na malilirip sa danas ng mga deboto.

\section{Sanggunian}

Bautista, J. (2010). Figuring catholicism: AneEthnohistory of the Santo Niño de Cebu. Quezon City: Ateneo de Manila University Press.

Bautista, J. (2014). Figuring catholicism: An ethnohistory of the Santo Niño de Cebu. Philippine Studies: Historical and Ethnographic Viewpoints, 62(3-4), 579-583.

Bautista, J. (2021). On the personhood of sacred objects: Agency, materiality and popular devotion in the roman catholic Philippines. Religions.

Beckett, J. (2015). Obligations of religion. Retreived March 24, 2018 from http://www.franksonnenbergonline.com/blog/7

Braun, V., \& Clarke, V. (2006). Using thematic analysis in psychology. Qualitative Research in Psychology, 3(2), 77-101.

Brion, M., Glorie Ann C., Diana M., Nedissa L., \& Ebora L. (2018). Devotees’ testimonies of faith of Señor Santo Niño De Cebu. Asia Pacific Journal of Multidisciplinary Research, 6, 89-96.

Christy, B. (2010). Ivory worship. National Geographic Magazine. https://www.nationalgeographic.com/ magazine/2012/10/blood-ivory/

Covar, P. (1998). Kaalamang bayang dalumat ng pagkataong pilipino. Sa kanyang Larangan: Seminal Essays on 
Philippine Culture. Maynila: NCCA, 9-19.

De Mesa, J. (1987). In solidarity with the culture: Studies in theological re-Rooting. Quezon City: Maryhill School of Theology.

Del Castillo, F., \& Sarmiento, P. J. (2021). A systematic review on spiritual modeling: Opportunities and challenges in religious education. International Journal of Christianity \& Education https://doi.org/10.1177/20569971211038968

Del Castillo, F., del Castillo, C. D., \& Corpuz, J. C. (2021). Dungaw: Re-imagined Religious Expressions in Response to the COVID-19 Pandemic. J Religion Health 60, 2285-2305.

Enriquez, V. (1993). Developing a filipino psychology. In Uichol Kim and John Berry (Eds.), Indigenous Psychologies: Research and Experience in Cultural Context (pp. 152-169). Sage.

Fabrega, M. (2009). How gratitude change your life. Retreived March 23, 2018 from http://.thechangeblog.com/gratitude/

Gonzales, L. (1976). Ang pagtatanung-Tanong: Dahilan at katangian. In Rogelia Pe-Pua (Ed.), Sikolohiyang Pilipino: Teorya, Metodo at Gamit (pp. 175-186). Lungsod Quezon: Surian ng Sikolohiyang Pilipino.

Hornborg, A. (2006). Animism, fetishism, and objectivism as strategies for knowing (or Not Knowing) the World. Ethnos, 71, 21-32.

Lyubomirsky, S. (2008). Expressing gratitude. Retreived March 23, 2018 from http://gratefulness.org/resource/ expressing_gratitude

Mercado, L. (1976). Elements of Filipino philosophy. Tacloban: Divine Word Publications.

Miranda, D. (1992). Buting Pinoy: Probe essays on value as Filipino. Manila: Divine Word Publications.

Pe-Pua, R., \& Protacio, E. (2000). Sikolohiyang pilipino: A legacy of virgilio G. enriquez. Asian Journal of Social Psychology, 3, 41-79.

Reyes, K. A., \& Bullecer, Ma. F. (2016). Karanasan at konsepto ng panata ng kapistahan ng ilang pamilyang pilipino sa lucban, quezon. The Bedan Journal of Psychology, 2.

Reynaldo I., Oliverio J., \& Ibones, N. (2016). Señor santo niño devotees' lived experiences in a fluvial parade. Asia Pacific Journal of Multidisciplinary Research, 4(2).

Roman Catholic Church. (1994). Catechism of the catholic church. Liguori: Liguori Publications.

Salazar, Z. (1990). Ethnic psychology and history: Reinterpreting faith healers. In V. G. Enriquez (Ed.), Indigenous psychology: A book of readings (p. 328). Quezon City: Akademya ng Kultura at Sikolohiyang Pilipino.

Whitehead, A. (2013). Religious statues and personhood: Testing the role of materiality. London: Bloomsbury Academic.

Yabut, H. (2013). Isang paglilinaw sa mga paniniwala at pagpapakahulugan sa ispiritwalidad at relihiyon ng mga pilipino. DIWA E-Journal Tomo I. DLSU, Manila Philippines. 